\title{
Effect of mineralocorticoid receptor antagonists on proteinuria and progression of chronic kidney disease: a systematic review and meta-analysis
}

Gemma Currie $^{1 *+}$ D, Alison H. M. Taylor ${ }^{1+}$, Toshiro Fujita ${ }^{2}$, Hiroshi Ohtsu ${ }^{3}$, Morten Lindhardt ${ }^{4}$, Peter Rossing ${ }^{4,5,6}$, Lene Boesby ${ }^{7}$, Nicola C. Edwards ${ }^{8}$, Charles J. Ferro ${ }^{8}$, Jonathan N. Townend ${ }^{8}$, Anton H. van den Meiracker ${ }^{9}$, Mohammad G. Saklayen ${ }^{10}$, Sonia Oveisi ${ }^{11}$, Alan G. Jardine ${ }^{1}$, Christian Delles ${ }^{1}$, David J. Preiss ${ }^{12}$ and Patrick B. Mark

\begin{abstract}
Background: Hypertension and proteinuria are critically involved in the progression of chronic kidney disease. Despite treatment with renin angiotensin system inhibition, kidney function declines in many patients. Aldosterone excess is a risk factor for progression of kidney disease. Hyperkalaemia is a concern with the use of mineralocorticoid receptor antagonists. We aimed to determine whether the renal protective benefits of mineralocorticoid antagonists outweigh the risk of hyperkalaemia associated with this treatment in patients with chronic kidney disease.

Methods: We conducted a meta-analysis investigating renoprotective effects and risk of hyperkalaemia in trials of mineralocorticoid receptor antagonists in chronic kidney disease. Trials were identified from MEDLINE (1966-2014), EMBASE (1947-2014) and the Cochrane Clinical Trials Database. Unpublished summary data were obtained from investigators. We included randomised controlled trials, and the first period of randomised cross over trials lasting $\geq 4$ weeks in adults.

Results: Nineteen trials (21 study groups, 1646 patients) were included. In random effects meta-analysis, addition of mineralocorticoid receptor antagonists to renin angiotensin system inhibition resulted in a reduction from baseline in systolic blood pressure $(-5.7[-9.0,-2.3] \mathrm{mmHg})$, diastolic blood pressure $(-1.7[-3.4,-0.1] \mathrm{mmHg})$ and glomerular filtration rate $\left(-3.2[-5.4,-1.0] \mathrm{mL} / \mathrm{min} / 1.73 \mathrm{~m}^{2}\right)$. Mineralocorticoid receptor antagonism reduced weighted mean protein/albumin excretion by $38.7 \%$ but with a threefold higher relative risk of withdrawing from the trial due to hyperkalaemia (3.21, [1.19, 8.71]). Death, cardiovascular events and hard renal end points were not reported in sufficient numbers to analyse.
\end{abstract}

Conclusions: Mineralocorticoid receptor antagonism reduces blood pressure and urinary protein/albumin excretion with a quantifiable risk of hyperkalaemia above predefined study upper limit.

\footnotetext{
* Correspondence: gemma.currie@glasgow.ac.uk

${ }^{\dagger}$ Equal contributors

${ }^{1}$ Institute of Cardiovascular and Medical Sciences, British Heart Foundation

Glasgow Cardiovascular Research Center, 126 University Place, Glasgow, UK

Full list of author information is available at the end of the article
} 


\section{Background}

Chronic kidney disease (CKD) is associated with risk of premature cardiovascular (CV) disease and death [1-6]. Hypertension (HTN) is the major modifiable risk factor for CKD progression and is associated with development of left ventricular hypertrophy (LVH) and proteinuria, both predictors of CV mortality [3, 7]. In CKD patients with proteinuria and/or HTN renin angiotensin system (RAS) inhibitors are commonly prescribed as these agents have been shown to reduce proteinuria and delay CKD progression through a combination of BP dependent and independent mechanisms. Despite this, patients still progress to end stage renal disease (ESRD) or die from CV events [8-14].

There is renewed interest in aldosterone as a mediator of $\mathrm{CV}$ and renal disease, beyond its BP effect resulting in an enthusiasm for using mineralocorticoid receptor antagonists (MRA) to minimised proteinuria and delay CKD progression. A 2009 meta-analysis, updated in 2014 demonstrated that addition of MRA to RAS blockade reduced $\mathrm{BP}$ and proteinuria in CKD $[15,16]$. The beneficial effects on outcomes were confounded by increased risk of hyperkalaemia, a factor limiting MRA prescribing in CKD [17-19]. Similar findings were described in a more recent metaanalysis on the cardiovascular actions of MRAs in CKD [20]. However, the conclusions of these analyses are drawn from mainly published proteinuria data only, derived by consolidating disparate urinary protein excretion measures used in different trials (variably reported as protein or albumin excretion in spot samples or $24 \mathrm{~h}$ collections). Furthermore, in some studies in these meta-analyses, the MRA effect is impossible to dissociate from that of additional antihypertensives co-administered with MRA in the intervention arm.

In the past 6 years several studies of effects of selective and non-selective MRAs in CKD have been published [21-34]. We performed an updated metaanalysis of this treatment strategy using summarised unpublished data where possible, as well as including data from 3 studies which were not considered in the previous publication [28, 30, 34]. This is particularly relevant as one of these studies focussed on patients with CKD stage 3-4 [30] an area where evidence for use of this strategy is lacking, and also because the resultant number of participants included exceeds that of the previous publications. We focused on change in urinary protein/albumin excretion, progression of CKD and risk of hyperkalaemia whilst additionally collecting hard clinical endpoints where these data were available. Our aim was to determine whether renoprotective benefits of MRAs outweigh risk of hyperkalaemia associated with this treatment.

\section{Methods}

Literature search was performed independently by two authors (GC, AT) using PubMed (1966 - 1st Dec 2014), EMBASE (1947 - 1st Dec 2014) and the Cochrane Clinical Trials Database. Search strategy is shown in Appendix 1 (see Additional file 1).

\section{Trial type}

We analysed randomised controlled trials in humans published in English of both selective and non-selective MRAs performed in CKD stage $1-5$ where MRA was compared to placebo or open label trials where MRA was additional therapy compared to the non-MRA arm. Trials were eligible if MRA was used alone or combined with ACE-I alone, ARB alone, or both ACE-I and ARB, performed in CKD patients or for prevention of CKD progression. The first period of randomised crossover trials was also considered eligible. Trials directly comparing MRA to other antihypertensive agents were excluded.

\section{Participants}

Trials enrolling patients with CKD stages 1-5 not requiring RRT, with albuminuria or proteinuria were included [11]. Our search included haemodialysis, peritoneal dialysis and renal transplantation ensure all appropriate trials were identified but RRT studies were excluded from the main analysis to minimise the confounding effect of dialysis on blood pressure and potassium.

\section{Interventions}

Trials using selective and non-selective MRAs with placebo, ACE-I, ARB or both were included. Minimum trial duration was 4 weeks.

\section{Outcome measures}

Analysis plan included effects of MRAs on the following measures:

a) End of treatment urinary albumin or protein excretion (24-h proteinuria or albuminuria, or urinary protein ratio or albumin creatinine ratio)

b) End of treatment renal function: serum creatinine $(\mu \mathrm{mol} / \mathrm{L})$; eGFR $\left(\mathrm{ml} / \mathrm{min} / 1.73 \mathrm{~m}^{2}\right)$; creatinine clearance $(\mathrm{ml} / \mathrm{min})$; doubling of serum creatinine; need for RRT. When several measures of kidney function were available this was meta-analysed following the hierarchy- isotopic GFR, creatinine clearance from 24 h urine collection, eGFR (MDRD or CKD-EPI formulae), formula estimated creatinine clearance (Cockcroft-Gault)

c) End of treatment SBP and DBP (mmHg) 
d) Hyperkalaemia (serum potassium above trial protocol limit)

e) Death, need for RRT, cardiovascular events.

\section{Data collection}

The search strategy in Additional file 1: Appendix 1 identified titles and abstracts. Both reviewers assessed titles and abstracts independently, discarding those not meeting inclusion criteria. Full texts of remaining trials were independently assessed. A third author (PM) settled discrepancies. Data extraction was performed using specific extraction forms (Appendix 2, see Additional file 1). Original authors were contacted tor further information.

\section{Assessment of risk of bias}

Two independent reviewers (GC, AT) assessed trial quality using the Cochrane Collaboration risk of bias assessment tool [35]. Items assessed were: adequate sequence generation; allocation concealment; blinding of participants, trial personnel and outcome assessors; reporting of incomplete outcome data; suggestion of selective outcome reporting and intention to treat analysis (Additional file 1: Table S1).

\section{Statistical analysis}

Random effects meta-analysis was performed for continuous and categorical outcomes. For continuous outcomes, weighted mean differences were performed using two approaches: in analyses of continuous data, final visit results were compared for treatment and control arms after verifying by meta-analysis that baseline data for the relevant outcome were no different between trial arms; second, where sufficient data were available, change in weighted mean difference from baseline to final visit was calculated by meta-analysis. For categorical outcomes, we calculated risk ratios (RRs) as ratio of cumulative incidence and $95 \%$ CIs from available data for trial participants at baseline and for those developing the outcome of interest. Random effects models were used as preferable approach to manage between-trial heterogeneity introduced by analysing differing trial populations. As standard deviations were unavailable for all measures of change in albuminuria and proteinuria, percentage change was analysed using weighted means and weighted standard deviations across trials in exploratory analyses. Statistical heterogeneity across studies was quantified using $\mathrm{I}^{2}$ statistics, providing measure of proportion of overall variation attributable to between-trial heterogeneity, with $p<0.10$ considered significant. We assessed publication bias with funnel plots and Egger tests, for the most commonly reported outcome SBP. Analyses were conducted using Stata version 13 (StataCorp, College Station, Texas).

\section{Results \\ Literature search and trial characteristics Search results}

Search of PubMed, EMBASE and the Cochrane database identified 299 citations. 243 were excluded (overlapping searches; non-randomised trials; trials evaluating interventions not included in this review) (Fig. 1). Full text assessment of 56 articles resulted in selection of 19 eligible trials including 1646 patients [22-31, 34, 36-43], more than were included in the previously published meta-analyses $[15,16,20]$. Authors were contacted for unpublished data; we obtained supplemental summarised results for ten trials [23, 26-30, 39, 40, 42, 43]. These were whole group mean and standard deviations pre- and post- intervention for the outcomes of interest, allowing a more complete and precise analysis.

One trial compared ACE-I and ARB (or placebo) and Spironolactone (or placebo), (four groups in total) [37] and another compared two doses of Eplerenone against placebo, (three groups in total) [38]. All comparable arms of both trials were included for analysis. Therefore, 19 trials of 21 study groups were included for analysis. Fourteen trials (889 patients) compared Spironolactone plus ACE-I or ARB with ACE-I or ARB alone; and 5 trials (757 patients) compared Eplerenone plus ACE-I or ARB to ACE-I or ARB alone. One trial focused on triple RAS blockade vs dual RAS blockade [41]. We did not identify any trials comparing MRA to placebo in the absence of ACE-I/ARB treatment.

\section{Trial characteristics}

Included were five randomised placebo controlled trials, seven randomised controlled trials (treatment compared to standard care) and seven randomised crossover trials. Six trials included patients with non-diabetic CKD, eight trials focused on diabetic nephropathy, 3 included both diabetic and non-diabetic CKD and 2 included patients with CKD and HTN.

Study duration was between 8 and 52 weeks. Sample size was small ( $n=18$ to 359$)$. No trials were powered to measure mortality or long-term renal outcomes. Dose of Spironolactone was $25 \mathrm{mg}$ /day in most trials, while two trials used $25-50 \mathrm{mg}[24,42]$. Eplerenone dose ranged from 25 to $100 \mathrm{mg}$.

The primary endpoint in the majority of trials was change in urinary protein/albumin excretion, although there was significant heterogeneity in measures used. Trials reported change in urine protein:creatinine ratio (PCR); albumin:creatinine ratio (ACR) or 24-h urine protein/albumin excretion. In three trials change in protein/albumin excretion was a secondary outcome measure where BP, pulse wave velocity and left ventricular mass index (LVMI) respectively were primary endpoints $[23,24,30]$. 


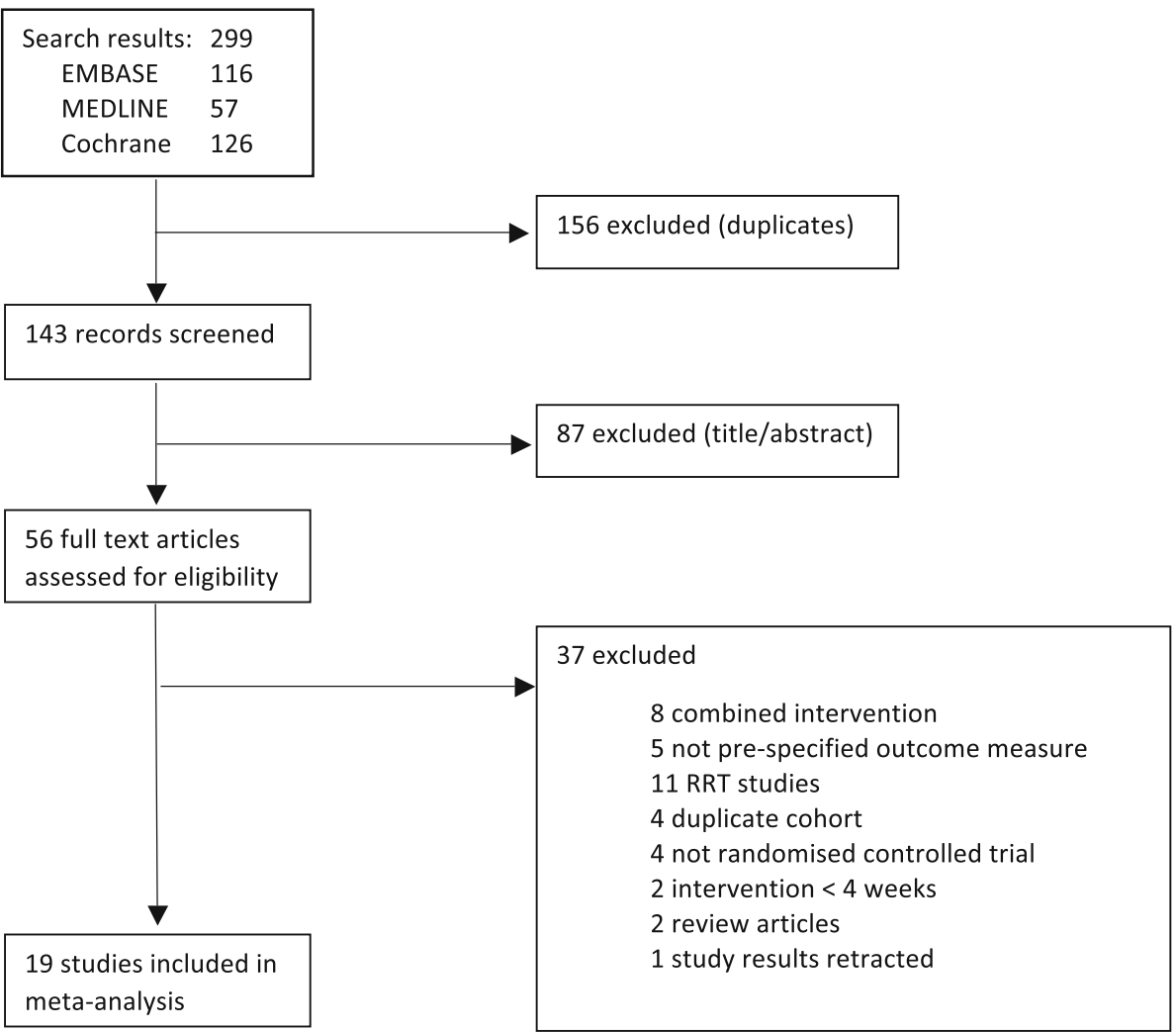

Fig. 1 Study flow chart

In trials reporting estimated glomerular filtration rate (eGFR) calculation method included Modification of Diet in Renal Disease (MDRD); Cockcroft Gault creatinine clearance $(\mathrm{CrCl})$ and Chronic Kidney Disease Epidemiology Collaboration (CKD-EPI) tools. In three trials GFR was measured by 51Cr-EDTA [26, 39, 40]. Characteristics of participants and interventions of included trials are listed in Table 1.

\section{Trial quality}

Trial quality assessed using the Cochrane Collaboration tool was variable (Additional file 1: Table S1). Sequence generation was adequately described in 9 (50 \%) trials. Allocation concealment was adequate in 8 (44 \%) trials. Participants and investigators were blinded in 12 (67 \%) trials and intention to treat analysis was performed in 2 (11 \%) trials. Dropouts were adequately accounted for in $16(84 \%)$ trials and did not differ between treatment and control groups.

\section{Trial outcomes}

Baseline data from included studies is shown in Additional file 1: Table S2. Meta-analysis of baseline data for all outcomes of interest showed these were balanced, confirming that end-of-trial meta-analysis was appropriate (Additional file 1: Table S3).

\section{Effect of treatment on blood pressure}

There was a significant change in systolic blood pressure (SBP) at final visit $(-5.7 \mathrm{mmHg})$ and in the nine trials where change from baseline $(-3.3 \mathrm{mmHg})$ was available, with addition of MRA to ACE-I and/or ARB in comparison to ACE-I and/or ARB alone (Fig. 2a). Addition of MRA to ACE-I and/or ARB also led to a significant change in end of trial diastolic blood pressure (DBP) $(-1.7 \mathrm{mmHg})$ and change from baseline to final visit $(-2.8 \mathrm{mmHg})$ in comparison to ACE-I and/or ARB alone (Table 2).

\section{Effect of treatment on renal excretory function}

There was a small, non-significant increase in end-oftrial serum creatinine $(3.8 \mu \mathrm{mol} / \mathrm{L})$ with addition of MRA to ACE-I and/or ARB compared to ACE-I and/or ARB alone (Table 3). Addition of MRA to ACE-I and/or ARB led to similar change in eGFR $(-2.7 \mathrm{~mL} / \mathrm{min} /$ $\left.1.73 \mathrm{~m}^{2}\right)$ and $\mathrm{CrCl}(-2.5 \mathrm{~mL} / \mathrm{min})$ compared to ACE-I and/or ARB alone with little heterogeneity between included study groups respectively $\left(\mathrm{I}^{2}=0 \%, P=0.696\right.$ and $\mathrm{I}^{2}=0 \%, P=0.727$ ) (Fig. 2b).

\section{Effect of treatment on urinary albumin/protein excretion}

In trials reporting $\mathrm{ACR}$, there was a non-significant change $(-10.91 \mathrm{mg} / \mathrm{mmol}$ creatinine) with addition of 
Table 1 Summary of populations and interventions in included studies. Data are mean \pm SD or median (IQR)

\begin{tabular}{|c|c|c|c|c|c|c|c|c|}
\hline Study & Kidney disease & $\begin{array}{l}\text { No. of patients } \\
\text { included }\end{array}$ & Intervention group & Control group & Co-intervention & $\begin{array}{l}\text { Study } \\
\text { duration }\end{array}$ & $\begin{array}{l}\text { Baseline eGFR } \\
\left(\mathrm{ml} / \mathrm{min} / 1.73 \mathrm{~m}^{2}\right)\end{array}$ & Endpoints \\
\hline Abolghasmi 2011 [24] & $\begin{array}{l}\text { CKD with resistant } \\
\text { hypertension }\end{array}$ & 41 & $\begin{array}{l}\text { Spironolactone } \\
25-50 \mathrm{mg}\end{array}$ & Placebo & $\begin{array}{l}\text { multi-drug regime } \\
\text { including ACE-I+/-ARB }\end{array}$ & 12 weeks & Not available & $\begin{array}{l}\text { BP, potassium, creatinine, } \\
\text { urinary sodium }\end{array}$ \\
\hline Ando 2014 [28] & CKD with hypertension & 314 & $\begin{array}{l}\text { Eplerenone } \\
50 \mathrm{mg}\end{array}$ & Placebo & $\begin{array}{l}\text { ACE-I+/-ARB of at least } \\
8 \text { weeks duration }\end{array}$ & 1 year & $\begin{array}{l}\text { Treatment } 67.7 \pm 14.3 \\
\text { Control } 68.6 \pm 13.6\end{array}$ & $\begin{array}{l}\text { UACR, creatinine, eGFR, } \\
\text { urinary L-FABP, } 24 \mathrm{~h} \\
\text { urinary sodium, incidence } \\
\text { of cerebrovascular and } \\
\text { cardiovascular events }\end{array}$ \\
\hline Bianchi 2006 [36] & $\begin{array}{l}\text { Non-diabetic CKD } \\
\text { (idiopathic GN) }\end{array}$ & 165 & $\begin{array}{l}\text { Spironolactone } \\
25 \mathrm{mg}\end{array}$ & ACE-I+/-ARB & ACE-I+/-ARB & 1 year & $\begin{array}{l}\text { Treatment } 62.4 \pm 21.9 \\
\text { Control } 62.2 \pm 19.0\end{array}$ & $\begin{array}{l}24 \mathrm{~h} \text { urinary protein, BP, } \\
\text { creatinine, eGFR potassium }\end{array}$ \\
\hline $\begin{array}{l}\text { Boesby } 2011[29] \\
(X O)\end{array}$ & Non-diabetic CKD & 40 & $\begin{array}{l}\text { Eplerenone } \\
25-50 \mathrm{mg}\end{array}$ & $\begin{array}{l}\text { multi-drug regime } \\
\text { including ACE-I+/-ARB }\end{array}$ & $\begin{array}{l}\text { multi-drug regime } \\
\text { including ACE-I+/-ARB }\end{array}$ & 8 weeks & $59 \pm 26$ & $\begin{array}{l}24 \text { h urinary albumin, BP, } \\
\text { potassium, creatinine } \\
\text { clearance }\end{array}$ \\
\hline Boesby 2013 [30] & $\begin{array}{l}\text { Diabetic and } \\
\text { non-diabetic CKD }\end{array}$ & 26 & $\begin{array}{l}\text { Eplerenone } \\
25-50 \mathrm{mg}\end{array}$ & ACE-I+/-ARB & ACE-I+/-ARB & 24 weeks & $36 \pm 10$ & $\begin{array}{l}\text { cfPWV, Alx, AASI, } 24 \text { h } \\
\text { urinary albumin }\end{array}$ \\
\hline $\begin{array}{l}\text { Chrysostomou } 2006^{\mathrm{a}} \\
\text { [37] }\end{array}$ & $\begin{array}{l}\text { Diabetic and } \\
\text { non-diabetic CKD }\end{array}$ & 41 & $\begin{array}{l}\text { Spironolactone } \\
25 \mathrm{mg}\end{array}$ & $\begin{array}{l}\text { Placebo as ARB; } \\
\text { Placebo as } \\
\text { Spironolactone }\end{array}$ & $\begin{array}{l}\text { ACE-I alone; ACE-I + } \\
\text { ARB }\end{array}$ & 3 months & Not available & $\begin{array}{l}24 \mathrm{~h} \text { urinary protein, } \mathrm{BP} \text {, } \\
\text { creatinine, creatinine } \\
\text { clearance, potassium }\end{array}$ \\
\hline Edwards 2009 [23] & $\begin{array}{l}\text { Non-diabetic CKD with } \\
\text { no renovascular } \\
\text { diagnosis }\end{array}$ & 112 & $\begin{array}{l}\text { Spironolactone } \\
25 \mathrm{mg}\end{array}$ & Placebo & ACE-I/ARB & 36 weeks & $\begin{array}{l}\text { Treatment } 49 \pm 12 \\
\text { Control } 53 \pm 11\end{array}$ & $\begin{array}{l}\text { LVMI, cfPWV, aortic } \\
\text { distensibility, Alx, BP }\end{array}$ \\
\hline Epstein 2006+ [38] & Diabetic nephropathy & 359 & $\begin{array}{l}\text { Eplerenone } \\
50 \mathrm{mg} \text { or } 100 \mathrm{mg}\end{array}$ & Placebo & ACE-I & 12 weeks & $\begin{array}{l}\text { ACE } \pm \text { EPL } 50 \\
73(62.1-83.6) \\
\text { ACE } \pm \text { EPL } 100 \\
75 \text { (62.8-85.9) } \\
\text { Control } \\
74(60.5-82.2)\end{array}$ & UACR, potassium, BP, eGFR \\
\hline Guney 2009 [25] & Non-diabetic CKD & 24 & $\begin{array}{l}\text { Spironolactone } \\
25 \mathrm{mg}\end{array}$ & ACE-I+/-ARB & ACE-I+/-ARB & 6 months & $\begin{array}{l}\text { Treatment } 63.0 \pm 22.71 \\
\text { Control } 56.3 \pm 35.6\end{array}$ & $\begin{array}{l}\text { UPCR, urinary TGF- } \beta 1 \text {, } \\
\text { eGFR, creatinine, } \\
\text { potassium, BP, aldosterone }\end{array}$ \\
\hline Mehdi 2009 [22] & Diabetic nephropathy & 81 & $\begin{array}{l}\text { Spironolactone } \\
25 \mathrm{mg}\end{array}$ & Placebo or ARB & ACE-I & 48 weeks & Not available & $\begin{array}{l}\text { UACR, BP, creatinine } \\
\text { clearance, potassium }\end{array}$ \\
\hline $\begin{array}{l}\text { Nielsen } 2012[26] \\
(X O)\end{array}$ & $\begin{array}{l}\text { Diabetes with } \\
\text { microalbuminuria }\end{array}$ & 21 & $\begin{array}{l}\text { Spironolactone } \\
25 \mathrm{mg}\end{array}$ & Placebo & ACE-I/ARB & 60 days & Not available & $\begin{array}{l}24 \text { h urinary albumin, } B P \text {, } \\
\text { GFR, urinary L-FABP, } \\
\text { urinary NGAL, urinary } \\
\text { KIM-1 }\end{array}$ \\
\hline $\begin{array}{l}\text { Rossing } 2005[39] \\
(X O)\end{array}$ & Diabetic nephropathy & 20 & $\begin{array}{l}\text { Spironolactone } \\
25 \mathrm{mg}\end{array}$ & Placebo & ACE-I+/-ARB & 8 weeks & Not available & $\begin{array}{l}24 \text { h urinary albumin, } \\
\text { BP, GFR }\end{array}$ \\
\hline $\begin{array}{l}\text { Saklayen } 2008[43] \\
(X O)\end{array}$ & Diabetic nephropathy & 24 & $\begin{array}{l}\text { Spironolactone } \\
25-50 \mathrm{mg}\end{array}$ & Placebo & ACE-I/ARB & 3 months & $\begin{array}{l}\text { Treatment } 61.9 \pm 23.4 \\
\text { Control } 54.4 \pm 20.1\end{array}$ & $\begin{array}{l}\text { BP, creatinine, potassium, } \\
\text { UPCR }\end{array}$ \\
\hline $\begin{array}{l}\text { Schjoedt } 2005[40] \\
(X O)\end{array}$ & Diabetic nephropathy & 20 & $\begin{array}{l}\text { Spironolactone } \\
25 \mathrm{mg}\end{array}$ & Placebo & ACE-I+/-ARB & 2 months & Not available & $\begin{array}{l}24 \text { h urinary albumin, } \\
\text { BP, GFR }\end{array}$ \\
\hline
\end{tabular}


Table 1 Summary of populations and interventions in included studies. Data are mean \pm SD or median (IQR) (Continued)

\begin{tabular}{|c|c|c|c|c|c|c|c|c|}
\hline $\begin{array}{l}\text { Tylicki } 2008[41] \\
(\mathrm{XO})\end{array}$ & Non-diabetic CKD & 18 & $\begin{array}{l}\text { Spironolactone } \\
25 \mathrm{mg}\end{array}$ & ACE-I + ARB & $A C E-I+A R B$ & 8 weeks & $107.8(93-140.9)$ & $\begin{array}{l}24 \text { h urinary protein, } B P \text {, } \\
\text { creatinine, potassium, } \\
\text { PRA, urinary NAG, urinary } \\
\text { PIIINP }\end{array}$ \\
\hline $\begin{array}{l}\text { Tylicki } 2012[31] \\
(\mathrm{XO})\end{array}$ & Non-diabetic CKD & 18 & $\begin{array}{l}\text { Eplerenone } \\
50 \mathrm{mg}\end{array}$ & ARB + Aliskiren & ARB & 8 weeks & Not available & $\begin{array}{l}\text { UACR, BP, creatinine } \\
\text { clearance, potassium }\end{array}$ \\
\hline $\begin{array}{l}\text { van den Meiracker } \\
2006 \text { [42] }\end{array}$ & Diabetic nephropathy & 53 & $\begin{array}{l}\text { Spironolactone } \\
25-50 \mathrm{mg}\end{array}$ & Placebo & ACE-I/ARB & 1 year & $\begin{array}{l}\text { Treatment } 93.1 \pm 45 \\
\text { Control } 66.3 \pm 35.1\end{array}$ & $\begin{array}{l}24 \mathrm{~h} \text { urinary protein, } \mathrm{BP}, \\
\text { creatinine, eGFR, potassium }\end{array}$ \\
\hline Wang 2013 [34] & $\begin{array}{l}\text { Diabetic and } \\
\text { non-diabetic CKD }\end{array}$ & 208 & $\begin{array}{l}\text { Spironolactone } \\
20 \mathrm{mg}\end{array}$ & $\begin{array}{l}\text { multi-drug regime } \\
\text { including ACE-I+/-ARB }\end{array}$ & $\begin{array}{l}\text { multi-drug regime } \\
\text { including ACE-I+/-ARB }\end{array}$ & 16 weeks & $\begin{array}{l}\text { Treatment } 65.8 \pm 22.2 \\
\text { Control } 66.5 \pm 24.3\end{array}$ & $\begin{array}{l}24 \mathrm{~h} \text { urinary protein, } \\
\text { creatinine, potassium, } \\
\text { eGFR, BP, aldosterone }\end{array}$ \\
\hline Ziaee 2013 [27] & $\begin{array}{l}\text { Diabetes with } \\
\text { microalbuminuria }\end{array}$ & 60 & $\begin{array}{l}\text { Spironolactone } \\
25 \mathrm{mg}\end{array}$ & ACE-I & ACE-I & 12 weeks & $\begin{array}{l}\text { Treatment } 79.8 \pm 18 \\
\text { Control } 82.5 \pm 19.1\end{array}$ & UACR, BP, potassium, eGFR \\
\hline
\end{tabular}

UACR urine albumin:creatinine ratio, UPCR urine protein:creatinine ratio, $A C E-$ angiotensin converting enzyme inhibitor, $A R B$ angiotensin receptor blocker, $C K D$ chronic kidney disease, $N G$ glomerulonephritis, $L-F A B P$ $U A C R$ urine albumin:creatinine ratio, $U P C R$ urine protein:creatinine ratio, $A C E-I$ angiotensin converting enzyme inhibitor, $A R B$ angiotensin receptor blocker, $C K D$ chronic kidney disease, $N G$ glomerulonephritis, $L-F A B P$
liver-type fatty acid binding protein, $X O$ crossover study design, $C P P W V$ carotid-femoral pulse wave velocity, $A I x$ augmentation index, $A A S I$ ambulatory arterial stiffness index, LVMI left ventricular mass index, TGF- $\beta 1$ transforming growth factor- $\beta 1, N G A L$ neutrophil gelatinase associated lipocalin, KIM-1 kidney injury molecule-1, PRA plasma renin activity, NAG n-acetyl- $\beta$-D-glucosaminidase, PIIINP amino-terminal propeptide of type III transforming growth factor- $\beta 1, N G A L$ neutrophil gelatinase associated lipocalin, KM-1 kidney injury molecule-1, PRA plasma renin activity, NAG n-acetyl- $\beta$-D-glucosaminidase, PIIINP amino-terminal propeptide of type II procollagen, athis study had 4 arms +this study had 3 arms 


\section{a}

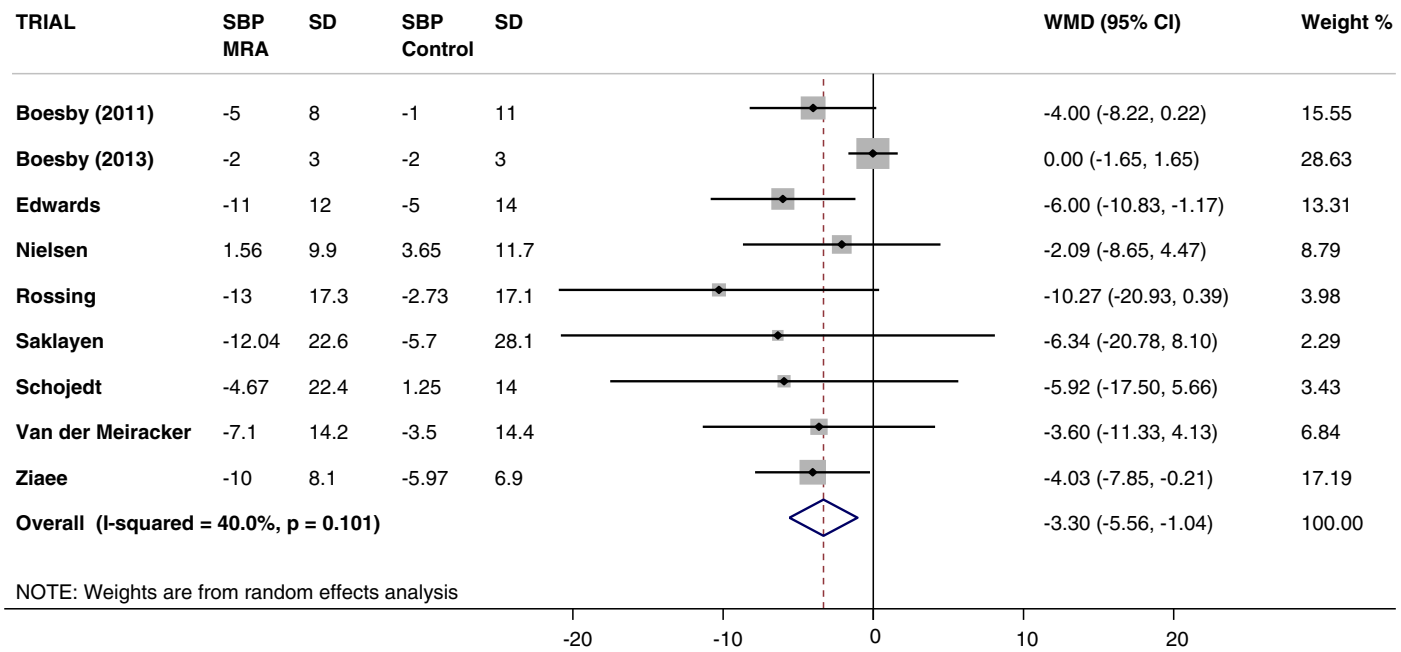

b

\begin{tabular}{|c|c|c|c|c|c|c|c|c|}
\hline TRIAL & $\begin{array}{l}\text { GFR } \\
\text { MRA }\end{array}$ & SD & $\begin{array}{l}\text { GFR } \\
\text { contro }\end{array}$ & SD & & & WMD (95\% Cl) & Weight $\%$ \\
\hline Ando & 64.1 & 17 & 68 & 17 & & & $-3.90(-7.56,-0.24)$ & 36.30 \\
\hline Bianchi & 58.6 & 24 & 56.4 & 21 & & & $2.20(-4.60,9.00)$ & 10.52 \\
\hline Boesby (2011) & 58 & 25 & 59 & 25 & & & $-1.00(-11.96,9.96)$ & 4.06 \\
\hline Boesby (2013) & 50 & 19 & 54 & 28 & $\rightarrow$ & & $-4.00(-17.18,9.18)$ & 2.80 \\
\hline Chrystosostomou A & 56.5 & 30 & 67.4 & 43 & $\begin{array}{c}1 \\
i\end{array}$ & & $-10.90(-43.09,21.29)$ & 0.47 \\
\hline Chrystosostomou B & 54.3 & 17 & 84.5 & 61 & & & $-30.20(-69.21,8.81)$ & 0.32 \\
\hline Edwards & 46 & 14 & 52 & 12 & $\rightarrow$ & & $-6.00(-10.83,-1.17)$ & 20.88 \\
\hline Guney & 58.4 & 24 & 58.7 & 39 & & & $-0.32(-23.53,22.89)$ & 0.90 \\
\hline Mehdi & 51.6 & 37 & 64.3 & 45 & & & $-12.70(-34.58,9.18)$ & 1.02 \\
\hline Nielsen & 68.8 & 31 & 75 & 30 & & & $-6.25(-24.83,12.33)$ & 1.41 \\
\hline Rossing & 71.4 & 28 & 74 & 26 & & & $-2.60(-19.18,13.98)$ & 1.77 \\
\hline Saklayen & 53.9 & 24 & 55.3 & 23 & & & $-1.32(-14.42,11.78)$ & 2.84 \\
\hline Schojedt & 81.3 & 26 & 84.7 & 29 & i- & & $-3.40(-20.26,13.46)$ & 1.71 \\
\hline Tylicki (2012) & 97.3 & 34 & 97.9 & 34 & 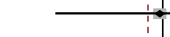 & & $-0.60(-23.05,21.85)$ & 0.97 \\
\hline Van der Meiracker & 75.2 & 37 & 59.8 & 35 & 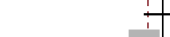 & & $15.40(-4.03,34.83)$ & 1.29 \\
\hline Ziaee & 75.6 & 16 & 79.6 & 17 & & & $-4.00(-12.33,4.33)$ & 7.02 \\
\hline Wang & 64.1 & 31 & 63.5 & 37 & $\overrightarrow{10}$ & & $0.60(-8.62,9.82)$ & 5.73 \\
\hline \multirow{2}{*}{\multicolumn{6}{|c|}{$\begin{array}{l}\text { Overall (l-squared }=\mathbf{0 . 0} \%, \mathbf{p}=\mathbf{0 . 7 9 0} \text { ) } \\
\text { NOTE: Weights are from random effects analysis }\end{array}$}} & & $-3.15(-5.36,-0.95)$ & 100.00 \\
\hline & & & & & & & & \\
\hline & & & & -60 & $\begin{array}{l}1 \\
-30\end{array}$ & 30 & 60 & \\
\hline
\end{tabular}

C

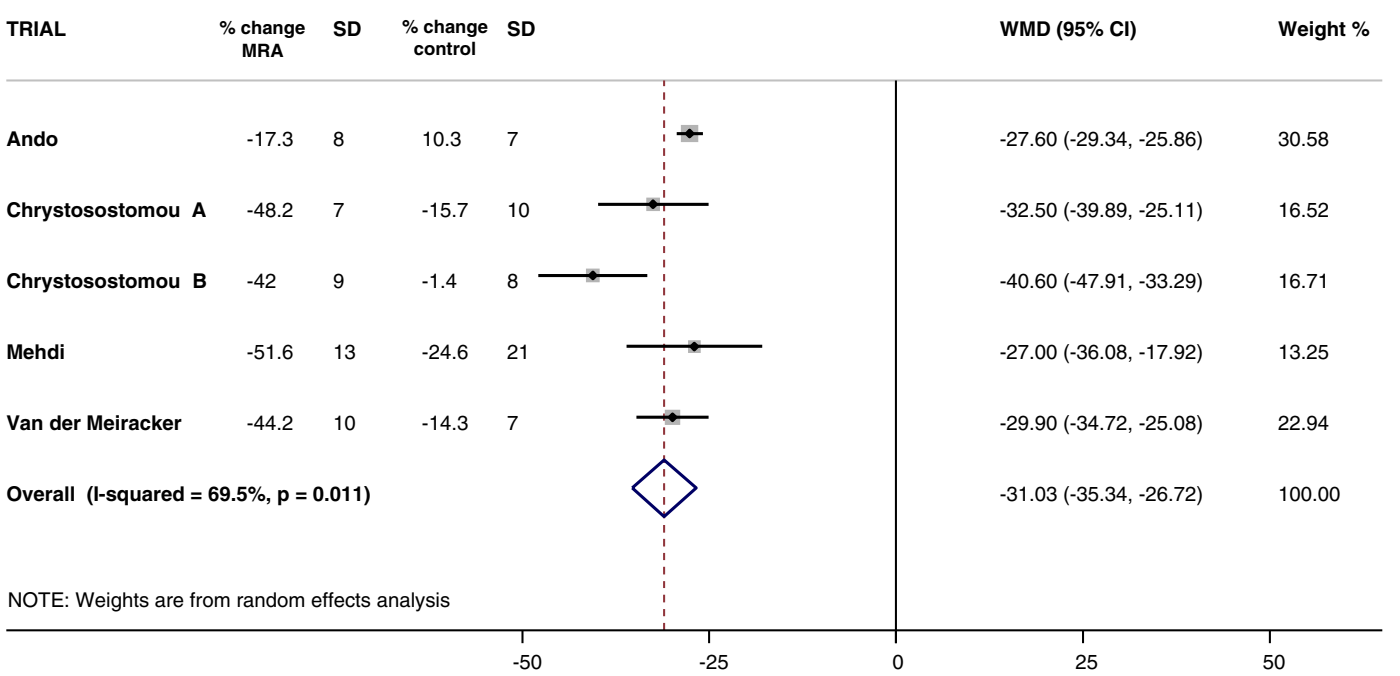

Fig. 2 (See legend on next page.) 
(See figure on previous page.)

Fig. 2 a Change in systolic blood pressure from baseline with addition of MRA to ACE-I and/or ARB compared to ACE-I and/or ARB alone. For participant numbers see Table 1. b Effect of the addition of MRA to ACE-I and/or ARB compared with ACE-I and/or ARB alone on end of treatment renal excretory function. For participant numbers see Table 1. c Percentage change from baseline of any measure of urinary protein/albumin excretion with the addition of MRA to ACE-I and/or ARB compared with ACE-I and/or ARB alone. For participant numbers see Table 1

MRA to ACE-I and/or ARB (Table 3). Where data for end-of-trial 24-h urinary albumin excretion were available, there was significant change associated with MRA in addition to ACE-I and/or ARB ( $-332.9 \mathrm{mg} / 24 \mathrm{~h})$. This was confirmed in the 3 trials reporting change from baseline albumin excretion, $(-292.23[-422.2,-162.3]$ $\mathrm{mg} / 24 \mathrm{~h}$ ). In trials reporting PCR, MRA added to ACE-I and/or ARB led to significant change in end-of-trial values $(-0.91 \mathrm{~g} / \mathrm{g}$ creatinine) compared with ACE-I and/ or ARB alone (Table 3). Change in 24-h urine protein from baseline to end-of-trial was available from two trials and showed a trend towards reduction $(-0.41 \mathrm{~g} / 24 \mathrm{~h})$ (Table 3) [34, 41].

Analysis of absolute values may be misleading with non-parametric data; therefore we assessed relative change from baseline in urinary protein/albumin excretion where these data were available. There was a significant reduction in urinary protein/albumin excretion with MRA analysed using the 5 trials which reported percentage change from baseline and where SD were available (Fig. 2c); and also when analysed as percentage change in any measure of urinary protein/albumin excretion. Using difference in means analysis in order to include data from all 19 trials, addition of MRA to ACE-I and/or ARB led to weighted mean reduction of $38.7 \%$ (weighted SD $21.5 \%$ ) in any measure of protein/ albumin excretion. The pattern of association between reduction in SBP and percentage reduction in urinary protein/albumin excretion is shown in Additional file 1: Figure S1.

\section{Effect of treatment on potassium}

Addition of MRA to ACE-I and/or ARB led to a moderate increase from baseline potassium compared to ACE-I and/or ARB alone both at end-of-trial visit $(0.19 \mathrm{mmol} / \mathrm{L}$ [95 \% CI 0.07-0.31]; 16 trials; $n=1356$;
$\left.\mathrm{I}^{2}=83.8 \%\right)$ and analysed as change from baseline to end-of-trial $(0.19 \mathrm{mmol} / \mathrm{L}$ [95 \% CI 0.12-0.27]; 8 trials; $n=478 ; \mathrm{I}^{2}=0 \%$. Baseline serum creatinine had no impact on difference in end of trial potassium $(p=0.15)$ or risk of hyperkalaemia $(p=0.21)$.

MRA was associated with threefold increased risk of hyperkalaemia above the predefined trial limit (Fig. 3a). Diabetic CKD patients were not at greater risk of developing hyperkalaemia than patients with CKD of alternative aetiology $(p=0.38)$ (Fig. $3 \mathrm{~b})$. Number needed to harm for 1 year of treatment, calculated from trials reporting at least one case of hyperkalaemia, was 10 (95 \% CI 5-27). Relative risk of being withdrawn from active treatment due to hyperkalaemia was increased (RR 3.21, 95 \% CI 1.19, 8.71), equating to number needed to harm over 1 year of 23 (95\% CI 7-267) in trials where at least one patient stopped therapy.

\section{Hard clinical endpoints}

Included studies were not adequately powered for analysis of hard clinical outcomes. No studies reported participants commencing RRT during the course of the trial. One study reported a participant death (cause unknown) in the treatment group [28] but there were no deaths reported from the remaining 18 studies. Cardiovascular morbidity was reported by two groups $[22,28]$. In one study, one patient in the treatment group developed atrial fibrillation and one in the control group suffered a cerebrovascular accident (CVA) [28]. Mehdi et al. reported occurrence of two CVA, two hospitalisations for heart failure, one myocardial infarction and one coronary artery bypass graft in the treatment group; whilst one subject in the placebo group suffered a CVA (Additional file 1: Table S4) [22]. A significant reduction in mortality with addition of MRA was seen in dialysis studies (Additional file 1: Figure S2).

Table 2 Effect of addition of MRA on final visit blood pressure

\begin{tabular}{llllllr}
\hline Variable & Measurement & $\begin{array}{l}\text { No. of study } \\
\text { groups }\end{array}$ & $\begin{array}{l}\text { No. patients in } \\
\text { intervention }\end{array}$ & $\begin{array}{l}\text { No. patients in } \\
\text { placebo/control }\end{array}$ & Effect size $(95 \% \mathrm{Cl})$ & $\mathrm{I}^{2}(p$ value $)$ \\
\hline Systolic BP (mmHg) & Change from baseline & 9 & 260 & 266 & $-3.30(-5.56,-1.04)$ & $40.0 \%(0.101)$ \\
& Final visit & 16 & 666 & 659 & $-5.69(-9.04,-2.34)$ & $81.8 \%(0.000)$ \\
Diastolic BP (mmHg) & Change from baseline & 9 & 260 & 266 & $-2.84(-3.35,-2.33)$ & $0.0 \%(0.799)$ \\
& Final visit & 16 & 666 & 659 & $-1.73(-3.37,-0.10)$ & $68.3 \%(0.000)$ \\
\hline
\end{tabular}


Table 3 Effect of addition of MRA on final visit renal function and urinary protein/albumin excretion

\begin{tabular}{|c|c|c|c|c|c|c|}
\hline Variable & Measurement & $\begin{array}{l}\text { No. of study } \\
\text { groups }\end{array}$ & $\begin{array}{l}\text { No. patients in } \\
\text { intervention }\end{array}$ & $\begin{array}{l}\text { No. patients in } \\
\text { placebo/control }\end{array}$ & Effect size (95 \% Cl) & $I^{2}$ ( $p$ value) \\
\hline Creatinine $(\mu \mathrm{mol} / \mathrm{L})$ & Final visit & 16 & 601 & 595 & $3.83(-2.14,9.79)$ & $50.4 \%(0.011)$ \\
\hline Creatinine Clearance (ml/min) & Final visit & 6 & 132 & 130 & $-2.51(-7.05,2.04)$ & $0.0 \%(0.599)$ \\
\hline eGFR (ml/min/1.73 m²) & Final visit & 13 & 626 & 617 & $-2.71(-4.85,-0.57)$ & $0.0 \%(0.727)$ \\
\hline GFR (any measure) & Final visit & 17 & 692 & 682 & $-3.15(-5.36,-0.95)$ & $0.0 \%(0.790)$ \\
\hline Urinary ACR (mg/mmol) & Final visit & 7 & 355 & 351 & $-10.91(-26.15,4.32)$ & $83.4 \%(0.000)$ \\
\hline Urinary PCR (g/g creatinine) & Final visit & 4 & 146 & 150 & $-0.91(-1.35,-0.46)$ & $58.4 \%(0.065)$ \\
\hline \multirow[t]{2}{*}{$24 \mathrm{~h}$ urinary albumin excretion (mg/24 h) } & Final visit & 6 & 151 & 155 & $-332.91(-624.80,-41.02)$ & $66.5 \%(0.011)$ \\
\hline & $\begin{array}{l}\text { Change from } \\
\text { baseline }\end{array}$ & 3 & 90 & 94 & $-292.23(-422.19,-162.27)$ & $0.0 \%(0.606)$ \\
\hline 24 h urinary protein excretion (g/24 h) & Final visit & 2 & 124 & 121 & $-0.41(-0.90,0.09)$ & $77.1 \%(0.037)$ \\
\hline
\end{tabular}

\section{Heterogeneity}

Heterogeneity was considerable for analyses of most outcomes (SBP, end-of-trial DBP, serum creatinine, urinary ACR and PCR, end-of-trial $24 \mathrm{~h}$ urinary albumin). However, heterogeneity was limited for other outcomes (Tables 2 and 3).

\section{Publication bias}

There was some suggestion of publication bias for SBP, evidenced by funnel plot (Fig. 4) and Egger test $(p=0.08)$, but not for end of study GFR or hyperkalaemia (Additional file 1: Figures S3 and S4).

\section{Discussion}

The beneficial impact of RAS blockade with ACE-I and ARB in both diabetic and non-diabetic CKD is well established [8-10, 13]. However, studies of combination therapy suggest harm in particular because of increased risk of hyperkalaemia and/or need for dialysis, and this treatment strategy is therefore generally avoided [44, 45]. Accumulating evidence suggests an independent role for aldosterone in development and progression of CV and renal disease [46-49]. Recent trials included in this analysis demonstrate beneficial effects on BP and urinary protein excretion in CKD with addition of MRA to ACE-I and ARB therapy. These benefits may however be offset by increased risk of hyperkalaemia or decline in renal function.

This meta-analysis confirms that MRA use in combination with ACE-I and/or ARB is associated with significant reductions in $\mathrm{BP}$ and end-of-treatment protein/ albumin excretion, while conferring a small and quantifiable increased risk of hyperkalaemia. These findings are in keeping with previous published meta-analyses $[15,16,20]$. By using additional unpublished summary results from a number of authors, we ensure that our protein/albumin excretion data are more comprehensive and hence more accurate. Through excluding studies where additional therapy was combined with MRA, we are confident that we report purely the effect of MRA on outcomes. Furthermore, we report clinically relevant risk of hyperkalaemia as relative risk and number needed to harm.

Many studies show strong independent associations between albuminuria and development of ESRD, translating into its widespread use as a surrogate outcome in CKD trials. A recent meta-analysis of over 78000 patients confirmed that $30 \%$ reduction in albuminuria confers 23.7 \% reduction in risk of progression to ESRD, irrespective of drug class [50]. We demonstrate that addition of MRA to ACE-I and/or ARB achieves $40 \%$ reduction in measures of protein/albumin excretion. This should translate to greater benefits in reduction in risk of ESRD and potentially in CV disease [51]. It is possible that reduction in protein/albumin excretion seen with MRA treatment is not entirely BP dependent (Additional file 1: Figure S1). $\mathrm{BP}$ independent effects of MRA on proteinuria are difficult to determine in the presence of substantial BP lowering effects with MRA seen across the trials.

Follow-up period of included trials was $<1$ year and mean baseline eGFR was $>35 \mathrm{ml} / \mathrm{min} / 1.73 \mathrm{~m}^{2}$ in all studies, therefore impact of addition of MRA to RAS blockade on longer-term renal outcomes or mortality in the later stages of CKD cannot be evaluated. Establishing efficacy of MRA treatment on morbidity and mortality in CKD requires longer follow up and larger sample size. One ongoing study may address this [52]. None of the studies included were powered to detect differences in hard renal end-points, CV morbidity or mortality. We cannot draw conclusions regarding longer-term safety and efficacy of combination MRA and/or ARB, despite postulated $\mathrm{CV}$ benefits of reducing protein/albumin excretion [53]. This is in keeping with a meta-analysis published last year [20].

Hyperkalaemia is an inherent risk associated with using MRA combined with RAS blockers and a common 


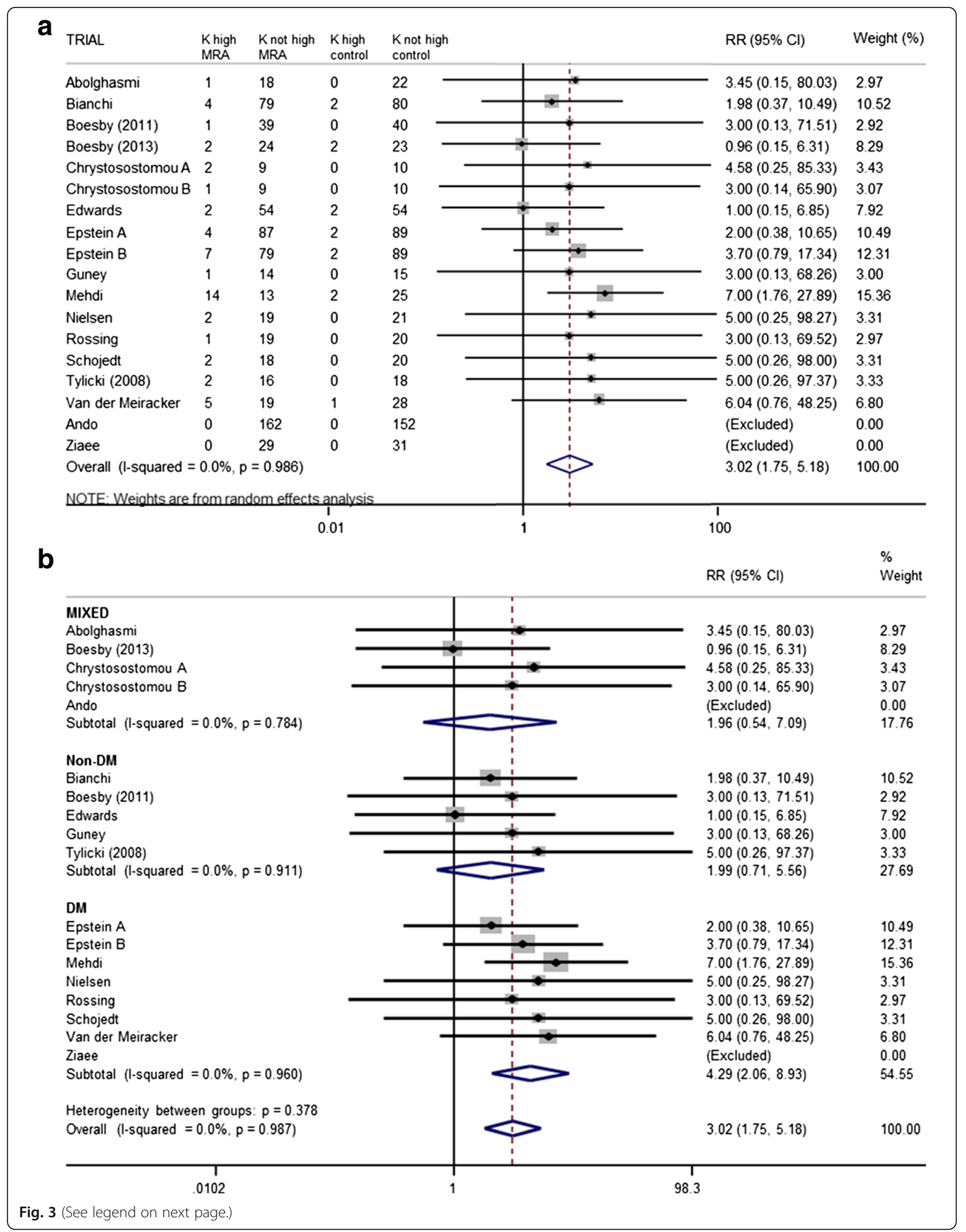


(See figure on previous page.)

Fig. 3 a Relative risk of developing hyperkalaemia above the predefined study limit with the addition of MRA to ACE-I and/or ARB compared to ACE-I and/or ARB alone. For participant numbers see Table 1. b Relative risk of developing hyperkalaemia above the predefined study limit with the addition of MRA to ACE-I and/or ARB compared to ACE-I and/or ARB alone based on aetiology of CKD (DM diabetes mellitus) included in trial. For participant numbers see Table 1

occurrence in studies of dual RAS blockade. Data from the ONTARGET study showed that combination ACE-I and ARB increased risk of dialysis, doubling of creatinine or death and combination treatment is not recommended in clinical guidelines [44]. We show an average potassium increase of $0.19 \mathrm{mmol} / \mathrm{L}$ at end of treatment (similar to previous data $[15,16]$ ) and three times greater risk of hyperkalaemia in patients receiving MRA in addition to ACE-I and/or ARB. It is essential to consider that this does not necessarily equate to risk of developing clinically significant hyperkalaemia requiring treatment, rather of developing a serum potassium level above the predefined study upper limit, which in many trials was $5.5-6 \mathrm{mmol} / \mathrm{L}$. Although few patients were withdrawn due to hyperkalaemia, many trials reported no withdrawals and only one study reported a single incidence of hyperkalaemia requiring hospital admission for treatment. The implications of these findings are unclear but the small increase in potassium suggests that if patients with high-normal baseline potassium values are excluded from treatment, the risk of dangerous hyperkalaemia may in fact be low. Hyperkalaemia has been associated with risk of renal events in patients with diabetes and nephropathy [54]. Our analysis did not demonstrate diabetic CKD to be at greater risk of developing hyperkalaemia than non-diabetic CKD.
Patients prescribed MRA require regular monitoring of potassium. Although serum potassium levels climb with chronic dosing in dual RAS blockade, the pattern is of early increase followed by steady state, not continuous increase [19]. It is conceivable that benefits conferred by $40 \%$ reduction in proteinuria outweigh the risk of small rises in potassium. Recent data suggest that nonsteroidal mineralocorticoid receptor antagonists may carry a reduced risk of side-effects, and these agents have shown promise for reduction of albuminuria in diabetic CKD [55]. In addition, recent literature highlights the potential for use of potassium binders in higher risk patients [56]. As well as addressing safety, evaluation of economic effects of increased monitoring of potassium compared reduction in $\mathrm{CV}$ events or progression of CKD is required.

Our study includes independent systematic searching, data extraction and assessment of study quality by two independent reviewers based on a pre-specified strategy. In addition, many authors provided supplemental summary data thereby enabling more comprehensive analysis. Furthermore, we obtained data from 3 studies which were not included in the previous meta-analyses $[28,30,34]$, resulting in analysis of data from a higher total number of patients (1646 vs 1549). This metaanalysis has several limitations. The majority of studies

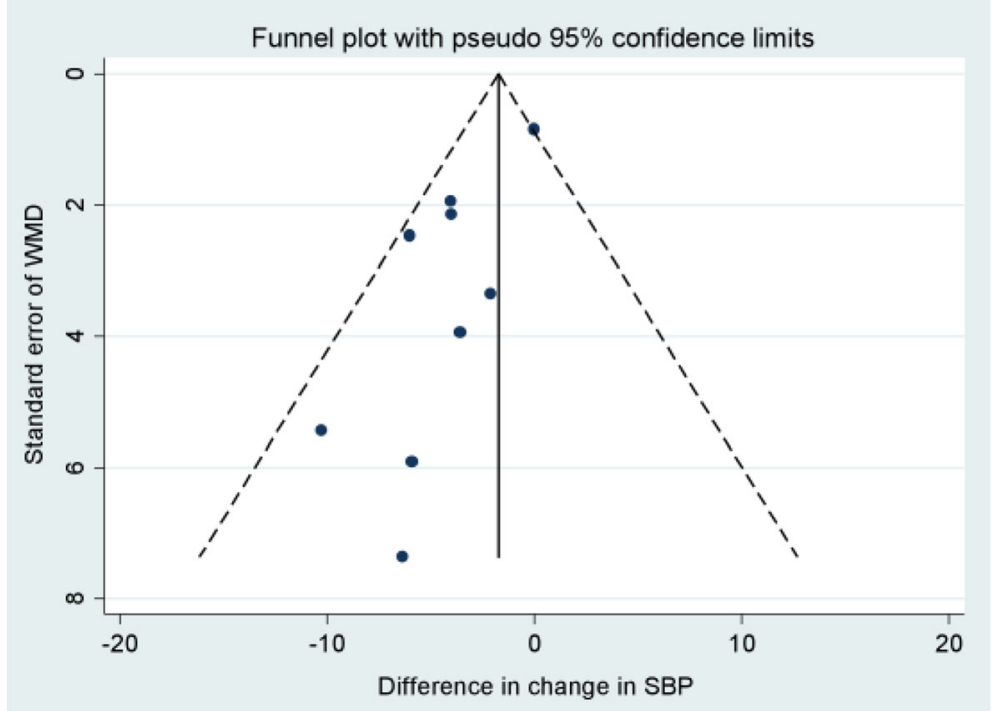

Fig. 4 Funnel plot (pseudo $95 \%$ confidence limits) demonstrating some evidence of publication bias for SBP (Egger test $p=0.08$ ) 
included patients with CKD stages 1-3 and therefore results may not be readily transferrable to populations with more advanced renal disease. There is a lack of long-term follow up data on clinical endpoints such as mortality and CKD progression. Studies included were small and powered to detect differences in surrogate endpoints. Seven included studies had crossover design [26, 29, 31, 39-41, 43] and reporting of methodology was variable. Adequate assessment of trial quality was not possible in all cases. There was significant heterogeneity in measurement of protein/albumin excretion. Authors reported ACR, PCR, $24 \mathrm{~h}$ albuminuria/proteinuria or combinations of these. Whilst the results demonstrate a significant reduction in protein or albumin excretion, this highlights that standardisation of measurements would allow consistent analysis, particularly if albuminuria (or equivalent) is used reliably as a surrogate for progression to ESRD [57].

\section{Conclusion}

In conclusion, in patients with CKD, with persistent proteinuria despite RAS inhibition, addition of MRA represents a promising treatment strategy for reducing BP and proteinuria with a quantifiable risk of hyperkalaemia. Appropriately designed larger studies with longerterm follow up are needed to address if MRA added to conventional RAS blockade reduces the risk of CV disease and need for RRT.

\section{Additional file}

Additional file 1: Table S1. Assessment of bias. Table S2. Baseline characteristics for individual study groups. Table S3. Comparison of baseline data in meta-analyses. Table S4. Events reported in trials analysed CVA- cerebrovascular accident; MI- myocardial infarction; CABG- coronary artery bypass graft. Figure S1. Plot of percentage reduction in proteinuria/albuminuria (any measure) SBP $(\mathrm{mmHg})$ at final visit across all studies. Each study is represented by a single circle, scaled to number of participants in the study. Figure S2. Effect of addition of MRA on all-cause mortality in RRT studies. Figure S3. Funnel plot (pseudo $95 \%$ confidence limits) showing no evidence of publication bias for GFR (Egger test $p=0.89$ ). Figure S4. Funnel plot (pseudo $95 \%$ confidence limits) showing no evidence of publication bias for hyperkalaemia (Egger test $p=0.81$ ). Appendix 1. Search strategy. Appendix 2. Sample data extraction form. (DOCX $192 \mathrm{~kb}$ )

\section{Funding}

Dr Taylor is funded by a Kidney Research UK Clinical Training Fellowship (Charity number 252892). Peter Rossing, Morten Lindhardt, Christian Delles, and Gemma Currie are funded by European Union FP7 Project PRIORITY (279277). The majority of trials discussed in this article were privately funded or were funded by non-pharmaceutical sources. Two trials were funded by Pfizer Inc $[28,38]$.

\section{Availability of data and materials}

This study includes summarised data collated from published trials listed in Table 1. No individual patient data was available.

\section{Authors' contributions}

Trial design (GC, AT), literature review (GC, AT, PM settled discrepancies), data collection \& collation of results (GC, AT), risk of bias (GC, AT), provision of summarised data (TF, HO, ML, PR, LB, NE, CF, JT, AM, MS, SO), statistical analysis (DP, GC, AT), preparation of manuscript (GC, AT, PM), review of manuscript (GC, AT, PM, CD, AJ, DP, TF, HO, ML, PR, LB, NE, CF, JT, AM, MS, SO). All authors read and approved the final manuscript.

\section{Competing interests}

The authors declare that they have no competing interests.

Consent for publication

Not applicable.

Ethics approval and consent to participate

Not applicable.

\section{Prior presentations/publication}

This work has been presented in abstract form at the American Heart Association Council on Hypertension and the Council on Kidney in Cardiovascular Disease Hypertension Scientific Sessions 2015. This work has not been published previously elsewhere.

\section{Author details}

'Institute of Cardiovascular and Medical Sciences, British Heart Foundation Glasgow Cardiovascular Research Center, 126 University Place, Glasgow, UK. ${ }^{2}$ Division of Clinical Epigenetics, Research Center for Advanced Science and Technology, The University of Tokyo, Tokyo, Japan. ${ }^{3}$ Department of Clinical Study and Informatics, Center for Clinical Sciences, National Center for Global Health and Medicine, Tokyo, Japan. ${ }^{4}$ Steno Diabetes Center, Niels Steensens Vej, Gentofte, Denmark. ${ }^{5}$ Health, Aarhus University, Aarhus, Denmark. ${ }^{6}$ NNF Centre for Basic Metabolic Research, University of Copenhagen, Copenhagen, Denmark. ${ }^{7}$ Department of Nephrology, Herlev Hospital, University of Copenhagen, Herlev, Denmark. ${ }^{8}$ Departments of Cardiology and Nephrology, University Hospital Birmingham and School of Clinical and Experimental Medicine, University of Birmingham, Birmingham, UK. ${ }^{9}$ Department of Internal Medicine and Pharmacy, Erasmus MC, Rotterdam, The Netherlands.

${ }^{10}$ VA Medical Center, 4100 West Third St, Dayton, OH 45428, USA.

${ }^{11}$ Metabolic Diseases Research Center, Qazvin University of Medical Sciences, Qazvin, Iran. ${ }^{12}$ Clinical Trial Service Unit and Epidemiological Studies Unit, University of Oxford, Oxford, UK.

Received: 23 December 2015 Accepted: 25 August 2016

Published online: 08 September 2016

\section{References}

1. Coresh J, Astor BC, Greene T, Eknoyan G, Levey AS. Prevalence of chronic kidney disease and decreased kidney function in the adult US population: Third National Health and Nutrition Examination Survey. Am J Kidney Dis. 2003;41:1-12.

2. Tonelli M, Wiebe N, Culleton B, House A, Rabbat C, Fok M, et al. Chronic kidney disease and mortality risk: a systematic review. J Am Soc Nephrol. 2006:17:2034-47.

3. Go AS, Chertow GM, Fan D, McCulloch CE, Hsu CY. Chronic kidney disease and the risks of death, cardiovascular events, and hospitalization. N Engl J Med. 2004;351:1296-305.

4. Tonelli M, Pfeffer MA. Kidney disease and cardiovascular risk. Annu Rev Med. 2007;58:123-39.

5. Coresh J, Selvin E, Stevens LA, Manzi J, Kusek JW, Eggers P, et al. Prevalence of chronic kidney disease in the United States. JAMA. 2007;298:2038-47.

6. Dor A, Pauly MV, Eichleay MA, Held PJ. End-stage renal disease and economic incentives: the International Study of Health Care Organization and Financing (ISHCOF). Int J Health Care Finance Econ. 2007;7:73-111.

7. McQuarrie EP, Freel EM, Mark PB, Fraser R, Patel RK, Dargie HG, et al. Urinary corticosteroid excretion predicts left ventricular mass and proteinuria in chronic kidney disease. Clin Sci (Lond). 2012;123:285-94.

8. Brenner BM, Cooper ME, de ZD, Keane WF, Mitch WE, Parving HH, et al. Effects of losartan on renal and cardiovascular outcomes in patients with type 2 diabetes and nephropathy. N Engl J Med. 2001;345:861-9.

9. Jafar TH, Schmid CH, Landa M, Giatras I, Toto R, Remuzzi G, et al. Angiotensinconverting enzyme inhibitors and progression of nondiabetic renal disease. A meta-analysis of patient-level data. Ann Intern Med. 2001;135:73-87. 
10. Jafar TH, Stark PC, Schmid CH, Landa M, Maschio G, de Jong PE, et al. Progression of chronic kidney disease: the role of blood pressure control, proteinuria, and angiotensin-converting enzyme inhibition: a patient-level meta-analysis. Ann Intern Med. 2003;139:244-52.

11. KDIGO. KDIGO 2012 clinical practice guideline for evaluation and management of chronic kidney disease. Kidney International Supplements. 2013;3:73-90

12. Tuttle KR, Bakris GL, Bilous RW, Chiang JL, de Boer $H$, Goldstein-Fuchs J, et al. Diabetic kidney disease: a report from an ADA consensus conference. Diabetes Care. 2014;37:2864-83.

13. Strippoli GF, Bonifati C, Craig M, Navaneethan SD, Craig JC. Angiotensin converting ensyme inhibitors and angiotensin $\|$ receptor antagonists for preventing the progression of diabetic kidney disease. Cochrane Database of Systematic Reviews Issue 4. 2014. 20-10-2014

14. Randomised placebo-controlled trial of effect of ramipril on decline in glomerular filtration rate and risk of terminal renal failure in proteinuric, non-diabetic nephropathy. The GISEN Group (Gruppo Italiano di Studi Epidemiologici in Nefrologia). Lancet. 1997;349:1857-63

15. Navaneethan SD, Nigwekar SU, Sehgal AR, Strippoli GF. Aldosterone antagonists for preventing the progression of chronic kidney disease: a systematic review and meta-analysis. Clin J Am Soc Nephrol. 2009;4:542-51.

16. Bolignano D, Palmer SC, Navaneethan SD, Strippoli GF. Aldosterone antagonists for preventing the progression of chronic kidney disease. Cochrane Database Syst Rev. 2014;4:CD007004.

17. Sica DA. Hyperkalemia risk in chronic kidney disease: deterrent to the use of aldosterone receptor antagonism or not. Hypertension. 2009;53:749-50.

18. Epstein M. Hyperkalemia as a constraint to therapy with combination ReninAngiotensin system blockade: the elephant in the room. J Clin Hypertens (Greenwich). 2009;11:55-60.

19. Preston RA, Afshartous D, Garg D, Medrano S, Alonso AB, Rodriguez R. Mechanisms of impaired potassium handling with dual renin-angiotensinaldosterone blockade in chronic kidney disease. Hypertension. 2009;53:754-60.

20. Ng K, Arnold J, Sharif A, Gill P, Townend J, Ferro C. Cardiovascular actions of mineralocorticoid receptor antagonists in patients with chronic kidney disease: a systematic review and meta-analysis. J Renin Angiotensin Aldosterone Syst. 2015;16:599-613.

21. Taheri S, Mortazavi M, Shahidi S, Pourmoghadas A, Garakyaraghi M, Seirafian $\mathrm{S}$, et al. Spironolactone in chronic hemodialysis patients improves cardiac function. Saudi J Kidney Dis Transpl. 2009;20:392-7.

22. Mehdi UF, Adams-Huet B, Raskin P, Vega GL, Toto RD. Addition of angiotensin receptor blockade or mineralocorticoid antagonism to maximal angiotensin-converting enzyme inhibition in diabetic nephropathy. J Am Soc Nephrol. 2009;20:2641-50.

23. Edwards NC, Steeds RP, Stewart PM, Ferro CJ, Townend JN. Effect of spironolactone on left ventricular mass and aortic stiffness in early-stage chronic kidney disease: a randomized controlled trial. J Am Coll Cardiol. 2009;54:505-12.

24. Abolghasmi R, Taziki O. Efficacy of low dose spironolactone in chronic kidney disease with resistant hypertension. Saudi J Kidney Dis Transpl. 2011; 22:75-8.

25. Guney I, Selcuk NY, Altintepe L, Atalay H, Basarali MK, Buyukbas S. Antifibrotic effects of aldosterone receptor blocker (spironolactone) in patients with chronic kidney disease. Ren Fail. 2009;31:779-84.

26. Nielsen S, Perrson F, Frandsen E, Sugaya T, Hess G, Zdunek D, et al. Spironolactone diminishes urinary albumin excretion in patients with type 1 diabetes and microalbuminuria: a randomized placebo-controlled crossover study. Diabet Med. 2012;29:e184-90.

27. Ziaee A, Abbas VA, Oveisi S, Javadi A, Hashemipour S, Kazemifar AM. Effects of additive therapy with spironolactone on albuminuria in diabetes mellitus: a pilot randomized clinical trial. Caspian J Intern Med. 2013;4:648-53.

28. Ando K, Ohtsu H, Uchida S, Kaname S, Arakawa Y, Fujita T. Anti-albuminuric effect of the aldosterone blocker eplerenone in non-diabetic hypertensive patients with albuminuria: a double-blind, randomised, placebo-controlled trial. Lancet Diabetes Endocrinol. 2014;2:944-53.

29. Boesby L, Elung-Jensen T, Klausen TW, Strandgaard S, Kamper AL. Moderate antiproteinuric effect of add-on aldosterone blockade with eplerenone in non-diabetic chronic kidney disease. A randomized cross-over study. PLoS One. 2011;6:e26904.

30. Boesby L, Elung-Jensen T, Strandgaard S, Kamper AL. Eplerenone attenuates pulse wave reflection in chronic kidney disease stage 3-4-a randomized controlled study. PLoS One. 2013;8:e64549.
31. Tylicki L, Lizakowski S, Rutkowski P, Renke M, Sulikowska B, Heleniak Z, et al. The enhanced renin-angiotensin-aldosteron system pharmacological blockade-which is the best? Kidney Blood Press Res. 2012;36:335-43.

32. Taheri S, Mortazavi M, Pourmoghadas A, Seyrafian S, Alipour Z, Karimi S. A prospective double-blind randomized placebo-controlled clinical trial to evaluate the safety and efficacy of spironolactone in patients with advanced congestive heart failure on continuous ambulatory peritoneal dialysis. Saudi J Kidney Dis Transpl. 2012;23:507-12.

33. Vukusich A, Kunstmann S, Varela C, Gainza D, Bravo S, Sepulveda D, et al. A randomized, double-blind, placebo-controlled trial of spironolactone on carotid intima-media thickness in nondiabetic hemodialysis patients. Clin J Am Soc Nephrol. 2010;5:1380-7.

34. Wang W, Li L, Zhou Z, Gao J, Sun Y. Effect of spironolactone combined with angiotensin converting enzyme inhibitors and/or angiotensin II receptor blockers on chronic glomerular disease. Experimental and Therapeutic Medicine. 2013;6:1527-31.

35. Higgins JP, Altman DG, Gotzsche PC, Juni P, Moher D, Oxman AD, et al. The Cochrane Collaboration's tool for assessing risk of bias in randomised trials. BMJ. 2011;343:d5928.

36. Bianchi S, Bigazzi R, Campese VM. Long-term effects of spironolactone on proteinuria and kidney function in patients with chronic kidney disease. Kidney Int. 2006;70:2116-23.

37. Chrysostomou A, Pedagogos E, MacGregor L, Becker GJ. Double-blind placebo-controlled study on the effect of the aldosterone receptor antagonist spironolactone in patients who have persistent proteinuria and are on long-term angiotensin-converting enzyme inhibitor therapy, with or without an angiotensin II receptor blocker. Clin J Am Soc Nephrol. 2006;1: 256-62.

38. Epstein M, Williams GH, Weinberger M, Lewin A, Krause S, Mukherjee R, et al. Selective aldosterone blockade with eplerenone reduces albuminuria in patients with type 2 diabetes. Clin J Am Soc Nephrol. 2006;1:940-51.

39. Rossing K, Schjoedt KJ, Smidt UM, Boomsma F, Parving HH. Beneficial effects of adding spironolactone to recommended antihypertensive treatment in diabetic nephropathy: a randomized, double-masked, cross-over study. Diabetes Care. 2005;28:2106-12.

40. Schjoedt KJ, Rossing K, Juhl TR, Boomsma F, Rossing P, Tarnow L, et al. Beneficial impact of spironolactone in diabetic nephropathy. Kidney Int. 2005;68:2829-36.

41. Tylicki L, Rutkowski P, Renke M, Larczynski W, Aleksandrowicz E, LysiakSzydlowska W, et al. Triple pharmacological blockade of the reninangiotensin-aldosterone system in nondiabetic CKD: an open-label crossover randomized controlled trial. Am J Kidney Dis. 2008;52:486-93.

42. Van Den Meiracker AH, Baggen RG, Pauli S, Lindemans A, Vulto AG, Poldermans D, et al. Spironolactone in type 2 diabetic nephropathy: Effects on proteinuria, blood pressure and renal function. J Hypertens. 2006;24: 2285-92.

43. Saklayen MG, Gyebi LK, Tasosa J, Yap J. Effects of additive therapy with spironolactone on proteinuria in diabetic patients already on ACE inhibitor or ARB therapy: results of a randomized, placebo-controlled, double-blind, crossover trial. J Investig Med. 2008;56:714-9.

44. The ONTARGET Investigators. Telmisartan, ramipril or both in aptients at high risk for vascular events. N Engl J Med. 2008;358:1547-59.

45. Fried LF, Emanuele N, Zhang JH, Brophy M, Conner TA, Duckworth W, et al. Combined angiotensin inhibition for the treatment of diabetic nephropathy N Engl J Med. 2013;369:1892-903.

46. Alfie J, Aparicio LS, Waisman GD. Current strategies to achieve further cardiac and renal protection through enhanced renin-angiotensinaldosterone system inhibition. Rev Recent Clin Trials. 2011;6:134-46.

47. Schjoedt KJ, Andersen S, Rossing P, Tarnow L, Parving HH. Aldosterone escape during blockade of the renin-angiotensin-aldosterone system in diabetic nephropathy is associated with enhanced decline in glomerular filtration rate. Diabetologia. 2004;47:1936-9.

48. Cerezo C, Ruilope LM, Segura J, Garcia-Donaire JA, de la Cruz JJ, Banegas JR, et al. Microalbuminuria breakthrough under chronic renin-angiotensinaldosterone system suppression. J Hypertens. 2012;30:204-9.

49. Aldigier JC, Kanjanbuch T, Ma LJ, Brown NJ, Fogo AB. Regression of existing glomerulosclerosis by inhibition of aldosterone. J Am Soc Nephrol. 2005;16: 3306-14.

50. Lambers-Heerspink HJ, et al. Drug-Induced Reduction in Albuminuria Is Associated with Subsequent Renoprotection: A Meta-Analysis. J Am Soc Nephrol. 2015;26(8):2055-64 
51. de ZD, Remuzzi G, Parving HH, Keane WF, Zhang Z, Shahinfar S, et al. Albuminuria, a therapeutic target for cardiovascular protection in type 2 diabetic patients with nephropathy. Circulation. 2004;110:921-7.

52. Hill NR, Lasserson D, Thompson B, Perera-Salazar R, Wolstenholme J, Bower $P$, et al. Benefits of Aldosterone Receptor Antagonism in Chronic Kidney Disease (BARACK D) trial-a multi-centre, prospective, randomised, open, blinded end-point, 36-month study of 2,616 patients within primary care with stage $3 \mathrm{~b}$ chronic kidney disease to compare the efficacy of spironolactone $25 \mathrm{mg}$ once daily in addition to routine care on mortality and cardiovascular outcomes versus routine care alone: study protocol for a randomized controlled trial. Trials. 2014;15:160

53. Matsushita $\mathrm{K}$, van $\mathrm{d} \mathrm{V}$, Astor $\mathrm{BC}$, Woodward M, Levey AS, de Jong PE, et al. Association of estimated glomerular filtration rate and albuminuria with allcause and cardiovascular mortality in general population cohorts: a collaborative meta-analysis. Lancet. 2010:375:2073-81.

54. Miao Y, Dobre D, Heerspink HJ, Brenner BM, Cooper ME, Parving HH, et al. Increased serum potassium affects renal outcomes: a post hoc analysis of the Reduction of Endpoints in NIDDM with the Angiotensin II Antagonist Losartan (RENAAL) trial. Diabetologia. 2011;54:44-50.

55. Bakris GL, Agarwal R, Chan JC, Cooper ME, Gansevoort RT, Haller H, et al. Effect of finerenone on albuminuria in patients with diabetic nephropathy: a randomized clinical trial. JAMA. 2015:314:884-94.

56. Weir MR, Bakris GL, Bushinsky DA, Mayo MR, Garza D, Stasiv Y, et al. Patiromer in patients with kidney disease and hyperkalemia receiving RAAS inhibitors. N Engl J Med. 2015;372:211-21.

57. Jun M, Turin TC, Woodward M, Perkovic V, Lambers Heerspink HJ, Manns BJ, et al. Assessing the validity of surrogate outcomes for ESRD: a meta-analysis. J Am Soc Nephrol. 2015

\section{Submit your next manuscript to BioMed Central and we will help you at every step:}

- We accept pre-submission inquiries

- Our selector tool helps you to find the most relevant journal

- We provide round the clock customer support

- Convenient online submission

- Thorough peer review

- Inclusion in PubMed and all major indexing services

- Maximum visibility for your research

Submit your manuscript at www.biomedcentral.com/submit 